\title{
Truncus Bicaroticus With Arteria Lusoria: A Rare Combination of Aortic Root Anatomy Complicating Cardiac Catheterization
}

\author{
Capt Roy Norris, MD, USAF, MC; CPT Andrew Wilson, DO, USA, MC; and MAJ Charles Lin, MD, USA, MC
}

\begin{abstract}
While most patients with arteria lusoria and common carotid trunk conditions are asymptomatic, discovery of such anomalies periprocedurally may affect the cardiac catheterization access site, catheter selection, and additional imaging.
\end{abstract}

\section{Roy Norris is a Cardiology Fellow in the Division of Cardiology, and Andrew Wilson is an Internal Medicine Resident, both at San Antonio Military Medical Center in Texas. Charles Lin is an Interventional Cardiologist deployed at William Beaumont Army Medical Center in El Paso, Texas. Correspondence: Roy Norris \\ (roy.s.norris2.mil@mail.mil)}

Fed Pract. 2021;38(2):84-89. doi:10.12788/fp.0089
B ranching of the great vessels from the aorta normally progresses with the brachiocephalic trunk as the first takeoff followed by the left common carotid and left subclavian artery in approximately 85\% of cases. ${ }^{1}$ Variants of great vessel branching patterns include the so-called bovine arch, arteria lusoria or aberrant right subclavian artery (ARSA), aberrant origin of the vertebral arteries, and truncus bicaroticus, or common origin of the carotid arteries (COCA). These aberrancies are quite rare, some with an incidence of $<1 \%$. ${ }^{1,2}$

These vascular anomalies become clinically relevant when they pose difficulty for operators in surgical and interventional specialties, necessitating unique approaches, catheters, and techniques to overcome. We present a case of concomitant aortic arch abnormalities during a diagnostic workup for transcatheter aortic valve replacement (TAVR) in a patient with previous coronary artery bypass grafting (CABG).

\section{CASE PRESENTATION}

A 66-year-old woman with coronary artery disease (CAD) status post-CABG and stage D1 aortic stenosis (AS) presented with exertional dyspnea. She was referred for coronary angiography as part of a workup for TAVR. Echocardiography confirmed severe AS with a peak velocity of $4.1 \mathrm{~m} / \mathrm{s}$, mean pressure gradient of $50 \mathrm{~mm} \mathrm{Hg}$, and an aortic valve area of $0.7 \mathrm{~cm}^{2}$. The patient was scheduled for cardiac catheterization with anticipated left radial artery approach for intubation and opacification of the left internal mammary artery (LIMA). However, this approach was abandoned during the procedure due to discovery of aberrant left radial artery anatomy, and the procedure was completed via femoral access.

Subsequent coronary angiography revealed 3-vessel CAD, patent saphenous vein grafts (SVG) to the right coronary artery (RCA) and a diagonal branch vessel with an occluded SVG to the left circumflex. Difficulty was encountered when engaging the left subclavian artery using a JR 4.0 diagnostic catheter for LIMA angiography. Nonselective angiography of the aortic arch was performed and demonstrated an uncommon anatomical variant (Figure 1, left). The right common carotid artery (CCA) $[\mathrm{A}]$ and the left CCA [B] arose from a single trunk, consistent with truncus bicaroticus or COCA [C]. The right subclavian artery [D] originated distal to the left subclavian artery otherwise known as arteria lusoria or ARSA forming an incomplete vascular ring [E]. Selective engagement of the left subclavian artery remained problematic even with the use of specialty arch catheters (Headhunter and LIMA catheters). The procedure concluded without confirming patency of the LIMA graft. A total of $145 \mathrm{~mL}$ of Omnipaque (iohexol injection) contrast was used for the procedure, and no adverse events occurred.

Same-day access of the ipsilateral ulnar artery was not pursued because of the risk of hand ischemia. The patient underwent repeat catheterization utilizing left ulnar artery access after adequate recovery time from the initial left radial approach. Selective LIMA 
angiography was achieved and demonstrated a patent LIMA to LAD graft. A computed tomography (CT) aorta for purposes of TAVR planning was able to reconstruct the aortic arch vasculature (Figure 1, right) confirming the presence of both ARSA and COCA. The patient went on to undergo successful TAVR with subsequent improvement of clinical symptoms.

\section{DISCUSSION}

Arteria lusoria is defined as an anomalous right subclavian artery arising distal to the origin of the left subclavian artery on the aortic arch. It has an estimated incidence of 0.5 to $2 \%$ and occurs as a consequence of abnormal embryologic involution of the right fourth aortic arch and right proximal dorsal aorta. This causes the origin of the right subclavian artery to shift onto the descending aorta and cross the mediastinum from left to right, passing behind the esophagus and the trachea. ${ }^{1,3-5}$

ARSA is often associated with other anatomic abnormalities, including COCA, rightsided aortic arch, interrupted aortic arch, aortic coarctation, tetralogy of Fallot, truncus arteriosus, transposition of the great arteries, atrial septal defects, and ventricular septal defects. Underlying genetic disorders, such as Edwards, Down, DiGeorge syndromes, aneurysms, and arterioesophageal fistulae can accompany these vascular malformations. ${ }^{6}$

COCA, such as we encountered, is the presence of a single branch from the aorta giving off both right and left common carotid arteries. It has an incidence of $<0.1 \%$ in isolation and is discovered most often in cadaveric dissections or incidentally on imaging. ${ }^{1}$ Its embryologic origin results from the third pair of cervical aortic arches persisting as a common bicarotid trunk. ${ }^{1,4,5}$ The combination of ARSA and COCA is rare. Of the 0.5 to $2 \%$ of ARSA cases discovered, only $20 \%$ of those cases present with associated COCA for a combined prevalence estimated at $<0.05 \%{ }^{7}$

The majority of patients with either anatomic abnormality are asymptomatic. However, a few classic clinical manifestations have been described. ARSA can rarely present with dysphagia lusoria, a condition resulting from an incomplete vascular ring formed by the abnormal course of the right
FIGURE 1 Angiograph (Left) and CT Reconstruction (Right)

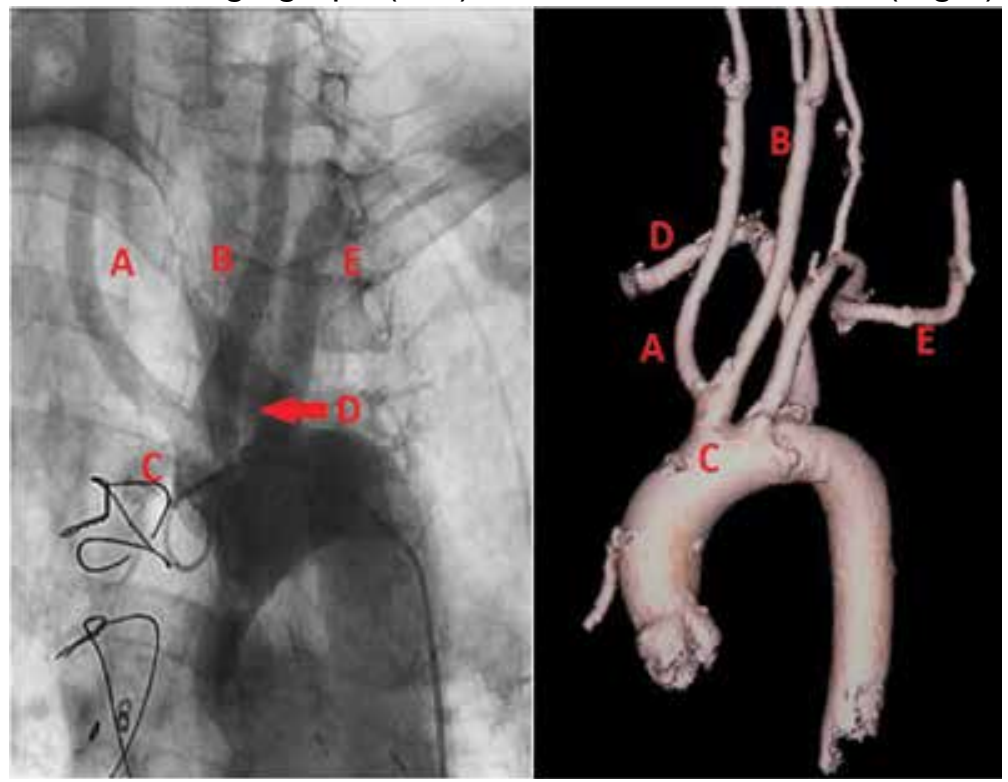

The right $\mathrm{CCA}[\mathrm{A}]$ and the left $\mathrm{CCA}[\mathrm{B}]$ arise from a common trunk (truncus bicaroticus) $[\mathrm{C}]$. The right subclavian artery [D] arises distal to the left subclavian artery (arteria lusoria) [E].

Abbreviations: CCA, common carotid artery; CT, computed tomography.

subclavian compressing the esophagus. Although not seen in our patient, it should be considered in the differential diagnosis for dysphagia. ${ }^{1,2,7}$ Ortner syndrome can result from right laryngeal nerve compression and palsy resultant from the aberrant course of the right subclavian artery. ${ }^{8}$ Another clinically relevant feature of ARSA is the presence of a diverticulum of Kommerell or dilatation at the origin of the right subclavian artery. It is a type of retroesophageal diverticulum resulting from persistence of a segment of the right sixth aortic arch. ${ }^{9}$ Finally, the spatial arrangement of ARSA increases risk for injury during head and neck surgical procedures, such as thyroidectomy, tracheotomy, and lymph node dissection of the right paratracheal fossa. ${ }^{6}$ Although the incidence is not well described, COCA has been described in several case reports as causing tracheal compression with dyspnea and in some cases, ischemic stroke. ${ }^{4,5,10}$

\section{Diagnosis}

The diagnosis of ARSA and COCA is often made incidentally on diagnostic imaging studies such as endovascular imaging, CT angiography, magnetic resonance (MR) angiography, postmortem cadaveric dissections, 
FIGURE 2 Adachi-Williams Classification of ARSA

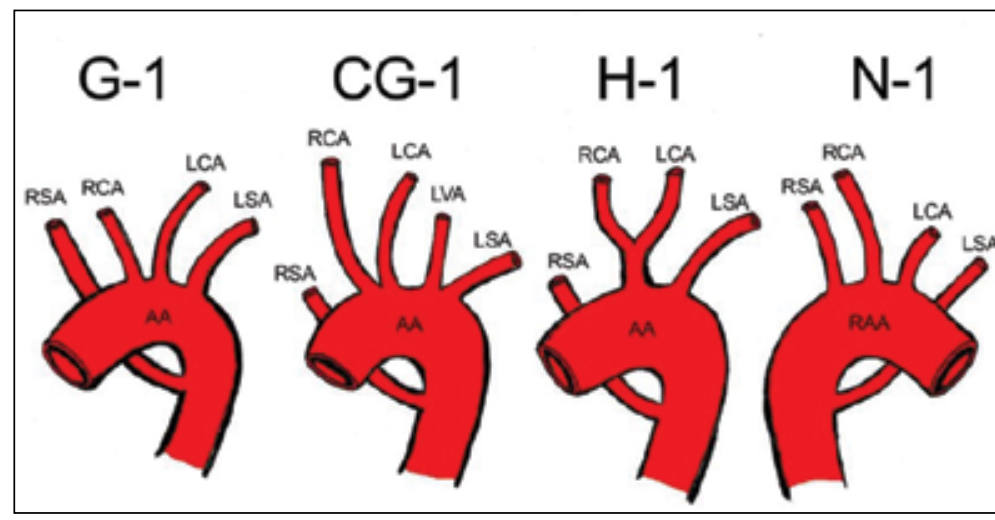

Abbreviations: AA, aortic arch; ARSA, aberrant right subclavian artery; LCA, left common carotid artery; LSA, left subclavian artery; LVA, left vertebral artery; RCA, right common carotid artery; RSA, right subclavian artery.

G-1, RSA originates from distal AA as last branch; CG-1, G-1 pattern with addition of LVA arising from $\mathrm{AA} ; \mathrm{H}-1, \mathrm{G}-1$ pattern with presence of common origin of the carotid arteries. $\mathrm{N}-1$, right-sided $A A$ with an aberrant LSA as most distal branch of $A A$ (mirror image of $G-1$ ). Reproduced with permission from Leite TFO, Pires LAS, Cisne R, Babinski MA, Chagas CAA. Clinical discussion of the arteria lusoria: a case report. J Vasc Bras. 2017;16(4):339-342. doi:10.1590/1677-5449.007617 radial artery anatomic variant was encountered in our case precluding left radial approach. In addition, abnormal takeoffs of the great vessels thwarted multiple attempts at intubation of the LSA (Figure 1, right). Some data suggest CT imaging can be of assistance in establishing patency of bypass grafts in CABG patients. ${ }^{15}$ This can be considered an option if branch-vessel anatomy remains unclear. Our patient exhibited several risk factors for stroke, including female gender, hypertension, and prior CABG. These and other risk factors may influence clinical decisions such as continued catheter manipulation, choice of catheter type, and further contrast studies. ${ }^{16}$

Nonselective angiography in these cases often can require excessive iodinated contrast, exposing the patient to increased risk of contrast-induced nephropathy (CIN). ${ }^{7,17}$ Although the amount of contrast used in our case was average for diagnostic catheterization, the patient went on to undergo a second catheterization and CT angiography to establish LIMA graft patency. ${ }^{17} \mathrm{CT}$ imaging reconstruction elucidated her aberrant branch-vessel anatomy. Patients are at increased risk of CIN with contrast loads $<200 \mathrm{~mL}$ per study, and this effect is compounded when the patient is elderly, has diabetes mellitus, and/or antecedent renal disease. ${ }^{18}$ Attention to the patient's preoperative glomerular filtration rate, avoidance of nephrotoxic agents, and intraoperative left ventricular end-diastolic pressure during cardiac catheterization with postcontrast administration of IV isotonic fluids have been shown to prevent CIN.$^{19,20}$ In the POSEIDON trial, fluid administration on a sliding scale based on the left ventricular end-diastolic pressure resulted in lower absolute risk of CIN postcatheterization vs standard postprocedure hydration in cardiac catheterization. ${ }^{21}$ Further, the now widespread use of low and iso-osmolar contrast agents further reduces the risk of CIN. ${ }^{22}$

For cardiac catheter laboratory operators, it is important to note that ARSA is more frequently encountered due to increased use of the transradial approach to coronary angiography. ${ }^{11}$ It should be suspected when accessing the ascending aorta proves exceptionally challenging and the catheter has a predilection for entering the descending aorta. ${ }^{11}$ 
While more technically demanding, 2 cases described by Allen and colleagues exhibited safe and successful entry into the ascending aorta with catheter rotation and hydrophilic support wires indicating the right radial approach is feasible despite presence of ARSA. ${ }^{12}$ Several patient-initiated maneuvers can be utilized to aid in accessing the ascending aorta. For example, deep inspiration to reduce the angulation between the aortic arch and ARSA. The use of curved catheters, such as Amplatz left, internal mammary catheter, or Simmons catheter may be considered to cannulate the ascending aorta if ARSA is encountered. Complications associated with a transradial approach include dissection and intramural hematoma. Minor bleeds and vasospasm also can occur secondary to increased procedural duration. ${ }^{6,8}$

\section{Treatment}

ARSA and COCA are considered normal anatomic variants and no treatment is indicated if the patient is asymptomatic. If symptoms are present, they often arise from aneurysmal or occlusive complications of the vascular anatomy. In patients with isolated ARSA and mild dysphasia or reflux symptoms, the use of prokinetics and antireflux medications may provide relief. It is important to note the coexistence of ARSA and COCA is more likely to produce esophageal compression compared to ARSA alone due to formation of a more complete vascular ring. Surgical management has been described in severe cases of ARSA involving risk of aneurysm rupture, right upper limb ischemia, or compression of the esophagus or trachea.

Several surgical approaches have been described, including simple ligation and division of ARSA and reimplantation of the RSA into the right CCA or ascending aorta. ${ }^{5}$ A recent review of 180 cases of ARSA diagnosed on CT angiography with concomitant common carotid trunk in half of studied individuals focused on a hybrid open and intravascular procedure. This procedure involved a double transposition or bypass (LSA to left common carotid artery and ARSA to the right CCA) followed by implantation of a thoracic stent graft. Few cases are eligible for these procedures or require them for definitive treatment. ${ }^{23}$

\section{CONCLUSIONS}

Recognition of aortic arch anatomical variants such as our case of ARSA with concomitant COCA may influence clinician decisions in various specialties, such as interventional cardiology, interventional neurology, cardiothoracic surgery, and gastroenterology. While most patients with these conditions are asymptomatic, some may present with dysphagia, dyspnea, and/or stroke symptoms. In our practice, discovery of such anomalies periprocedurally may affect cardiac catheterization access site, catheter selection, and additional imaging. The presence of arteria lusoria can be of critical importance when encountering a patient with myocardial infarction as switching from transradial to transfemoral approach may be required to gain access to the ascending aorta. Overall, transradial coronary angiography and percutaneous coronary intervention is not contraindicated in the setting of ARSA/COCA and can be safely performed by an experienced operator.

It is important for surgical specialists to be aware of the coexistence of anomalies where the discovery of one aberrancy can signal coexistent variant anatomy. If aortic arch anatomy is unclear, it is useful to perform nonselective angiography and/or further imaging with CT angiography. Knowledge of abnormal aortic arch anatomy can decrease fluoroscopy time and contrast load administered, thereby reducing potential periprocedural adverse events.

\section{Author disclosures}

The authors report no actual or potential conflicts of interest with regard to this article.

\section{Disclaimer}

The opinions expressed herein are those of the authors and do not necessarily reflect those of Federal Practitioner, Frontline Medical Communications Inc., the US Government, or any of its agencies.

\section{References}

1. Kurt MA, An I, Ikiz I. A case with coincidence of aberrant right subclavian artery and common origin of the carotid arteries. Ann Anat. 1997;179(2):175-176. doi:10.1016/s0940-9602(97)80100-8

2. Klinkhamer AC. Aberrant right subclavian artery. Clinical and roentgenologic aspects. Am J Roentgenol Radium Ther Nucl Med. 1966;97(2):438-446. doi:10.2214/ajr.97.2.438

3. Türkvatan A, Büyükbayraktar FG, Olçer T, Cumhur T. Congenital anomalies of the aortic arch: evaluation with the use of multidetector computed tomography. Korean $J$ Radiol. 2009;10(2):176-184. doi:10.3348/kjr.2009.10.2.176

4. Ozateş $M$, Nazaroglu $H$, Uyar $A$. MR angiography in di- 
agnosis of aberrant right subclavian artery associated with common carotid trunk. Eur Radiol. 2000;10(9):1503. doi: $10.1007 / \mathrm{s} 003300000335$

5. Poultsides GA, Lolis ED, Vasquez J, Drezner AD, Venieratos D. Common origins of carotid and subclavian arteria systems: report of a rare aortic arch variant. Ann Vasc Surg. 2004;18(5):597-600. doi:10.1007/s10016-004-0060-3

6. Leite TFO, Pires LAS, Cisne R, Babinski MA, Chagas CAA. Clinical discussion of the arteria lusoria: a case report. J Vasc Bras. 2017;16(4):339-342. doi:10.1590/1677-5449.007617

7. Tsai IC, Tzeng WS, Lee T, et al. Vertebral and carotid artery anomalies in patients with aberrant right subclavian arteries. Pediatr Radiol. 2007;37(10):1007-1012. doi:10.1007/s00247-007-0574-2

8. Rafiq A, Chutani S, Krim NR. Incidental finding of arteria lusoria during transradial coronary catheterization: significance in interventional cardiology. Catheter Cardiovasc Interv. 2018;91(7):1283-1286. doi:10.1002/ccd.27439

9. Priya S, Thomas R, Nagpal P, Sharma A, Steigner M. Congenital anomalies of the aortic arch. Cardiovasc Diagn Ther 2018;8(suppl 1):S26-S44. doi:10.21037/cdt.2017.10.15

10. Khatri R, Maud A, Rodriguez GJ. Aberrant right subclavian artery and common carotid trunk. J Vasc Interv Neurol. 2010;3(1):33-34.

11. Valsecchi O, Vassileva A, Musumeci G, et al. Failure of transradial approach during coronary interventions: anatomic considerations. Catheter Cardiovasc Interv. 2006;67(6):870-878. doi:10.1002/ccd.20732

12. Allen D, Bews $H$, Vo M, Kass M, Jassal DS, Ravandi A. Arteria lusoria: an anomalous finding during right transradial coronary intervention. Case Rep Cardiol. 2016;2016:8079856. doi:10.1155/2016/8079856

13. Fineschi M, ladanza A, Sinicropi G, Pierli C. Images in cardiology: angiographic evidence of aberrant right subclavian artery associated with common carotid trunk. Heart. 2002;88(2):158. doi:10.1136/heart.88.2.158

14. van Son JA, Julsrud PR, Hagler DJ, et al. Imaging strategies for vascular rings. Ann Thorac Surg. 1994;57(3):604610. doi:10.1016/0003-4975(94)90552-5

15. Lee R, Lim J, Kaw G, Wan G, Ng K, Ho KT. Comprehensive noninvasive evaluation of bypass grafts and na- tive coronary arteries in patients after coronary bypass surgery: accuracy of 64-slice multidetector computed tomography compared to invasive coronary angiography. J Cardiovasc Med (Hagerstown). 2010;11(2):81-90. doi:10.2459/JCM.0b013e32832f3e2e

16. Hamon M, Baron JC, Viader F, Hamon M. Periprocedura stroke and cardiac catheterization. Circulation. 2008;118(6): 678-683. doi:10.1161/CIRCULATIONAHA.108.784504

17. Hwang JR, D'Alfonso S, Kostuk WJ, et al. Contrast volume use in manual vs automated contrast injection systems for diagnostic coronary angiography and percutaneous coronary interventions. Can J Cardiol. 2013;29(3):372-376. doi:10.1016/j.cjca.2012.11.023

18. Rich MW, Crecelius CA. Incidence, risk factors, and clinical course of acute renal insufficiency after cardiac catheterization in patients 70 years of age or older. A prospective study. Arch Intern Med. 1990;150(6):1237-1242.

19. Davenport MS, Khalatbari S, Cohan $\mathrm{RH}$, Dillman JR, Myles JD, Ellis JH. Contrast material-induced nephrotoxicity and intravenous low-osmolality iodinated contrast material: risk stratification by using estimated glomerular filtration rate. Radiology. 2013;268(3):719728. doi:10.1148/radiol.13122276

20. American College of Radiology. ACR Manual on Contrast Media 2020. American College of Radiology; 2020:33-34. Accessed January 15, 2021. https://www.acr.org/-/media /ACR/Files/Clinical-Resources/Contrast_Media.pdf

21. Brar SS, Aharonian V, Mansukhani P, et al. Haemodynamic-guided fluid administration for the prevention of contrast-induced acute kidney injury: the POSEIDON randomised controlled trial. Lancet. 2014;383(9931):18141823. doi:10.1016/S0140-6736(14)60689-9

22. Aoun J, Nicolas D, Brown JR, Jaber BL. Maximum allowable contrast dose and prevention of acute kidney injury following cardiovascular procedures. Curr Opin Nephrol Hypertens. 2018;27(2):121-129. doi:10.1097/MNH.0000000000000389

23. Settembre N, Saba C, Bouziane Z, Jeannon F, Mandry D, Malikov S. Hybrid treatment of the aberrant right subclavian artery (arteria lusoria): feasibility study on 180 angio-CTs. Ann Vasc Surg. 2017;44:229-233. doi:10.1016/j.avsg.2017.03.172 